Vol. 2, No. 2, 2017

\title{
WIRELESS SENSOR-ACTUATOR NETWORK BASED ON THE SOC ESP8266 FOR MOBILE CYBER-PHYSICAL SYSTEMS
}

\author{
Alexey Botchkaryov, Yuriy Kraikin \\ Lviv Polytechnic National University, \\ Computer Engineering Department, 12, S. Bandera str., Lviv, 79013, Ukraine \\ Authors e-mail:alb2@ukr.net
}

Submitted on 03.12.2017

(C) Botchkaryov A., Kraikin Yu.. 2017

\begin{abstract}
The problem of wireless sensor-actuator network design as a part of the mobile cyber-physical system is considered. The ways of implementation of the wireless connections scheme are analyzed. The network structure and algorithms of its operation are proposed. The design of the sensor and actuator nodes based on the wireless SoC ESP8266 is presented.
\end{abstract}

Index Terms: wireless sensor-actuator network, cyberphysical system

\section{INTRODUCTION}

One of the main components of the modern cyberphysical systems (CPS) [1-5], including mobile CPS [6], is the wireless sensor-actuator network (WSAN) [7-9]. The number of different variants of practical use of WSANs and their scale is constantly increasing. Among the most prominent areas of practical use of WSANs one can distinguish home automation (smart houses), smart city technology, autonomous agricultural systems, autonomous transportation systems, autonomous systems of scientific research, etc. WSAN is an integral part of many mobile CPS built with the use of mobile computing technologies. It should also be noted that WSANs are one of the most promising areas for research, development and implementation of intelligent data collection technologies [10], including intelligent data collection technologies based on self-organization principles and the concept of structural adaptation [11-14].

In this paper we consider the problem of wireless sensor-actuator network design as a part of the mobile cyber-physical system. Various variants of WSAN node layout, various variants of wireless communication technologies for its construction and methods of implementation of the wireless communication scheme are analyzed. Also, the WSAN structure and algorithms of its operation based on the Wi-Fi technology are proposed in the paper. The design of the sensor and actuator nodes based on the wireless SoC ESP8266 is presented. The basic functionality of the designed WSAN includes: 1) the ability to collect measuring information from the sensors of different physical nature and execute commands by the actuators in automatic mode; 2) the ability to remotely view the sensor readings and give commands for the actuators from the control consoles on mobile computing devices.

\section{THE PROBLEM OF WSAN DESIGN}

Among the wireless technologies (standards) that can be used to design WSAN, one can consider the following most common wireless technologies for short distances: Bluetooth, ZigBee, and Wi-Fi. A comparison of these technologies and other most commonly used wireless technologies is presented in Table 1. For each of these technologies, there is a large variety of radio frequency modules of varying degrees of readiness that differ in technical parameters and characteristics. Therefore, the problem of developing a methodology for choosing a specific wireless communication technology and a specific element base (including wireless modules) for building a WSAN is particularly relevant.

In the context of this problem, the following tasks are solved in this paper: 1) a generalized WSAN scheme design, 2) analysis of various variants of the WSAN node layout, 3) analysis of various variants of implementation of the link scheme between WSAN components within the framework of the chosen layout variant, 4) selection of the node layout variant and wireless communication technology for the implementation of WSAN; 5) design of the WSAN structure and algorithms of its operation design; 6) design of sensor and actuator nodes of the network based on the chosen wireless communication technology.

\section{THE GENERALIZED SCHEME OF WSAN}

Considering the generalized WSAN scheme (Fig. 1), which includes: $\mathrm{S}$ - sensor node (collects measuring information from the sensors), A - actuator node (transforms and executes control commands), CU control unit (receives measuring information from sensor nodes, performs its analysis and generates control commands), $\mathrm{X}$ - control console (provides remote access to the control unit, for example from mobile computing device (smartphone)). 
Table 1

The main parameters of wireless technologies

\begin{tabular}{|c|c|c|c|c|c|c|}
\hline & ZigBee & Bluetooth & Wi-Fi & $434 / 868 \mathrm{MHz}$ & GSM/GPRS/EDGE & $3 G$ \\
\hline $\begin{array}{l}\text { Frequency range, } \\
\mathrm{MHz}\end{array}$ & $2400-2483$ & $2400-2483$ & $2400-2483$ & $434 / 868$ & $900 / 1800$ & $\begin{array}{l}\text { 1885-2025; } \\
\text { 2110-2200 }\end{array}$ \\
\hline Data rate, kbit/s & 250 & 721 & $11000 / 54000$ & 500 & $14,4 / 171 / 473$ & $144 / 384 / 2048$ \\
\hline Distance, $\mathrm{m}$ & 200 & $\begin{array}{l}\text { Class } 1-100 ; \\
\text { Class } 2-10 ; \\
\text { Class 3 - 1 }\end{array}$ & 100 & 1000 & coverage area & coverage area \\
\hline $\begin{array}{l}\text { Current } \\
\text { consumption, } \mathrm{mA}\end{array}$ & 30 & 70 & 450 & 30 & $350 / 3500$ & $350 / 3500$ \\
\hline Topology & $\begin{array}{l}\text { "point-to- } \\
\text { point", } \\
\text { "star", } \\
\text { network }\end{array}$ & $\begin{array}{l}\text { "point-to- } \\
\text { point", } \\
\text { "star", } \\
\text { network }\end{array}$ & $\begin{array}{l}\text { "point-to- } \\
\text { point", } \\
\text { "star" }\end{array}$ & $\begin{array}{l}\text { "point-to- } \\
\text { point", } \\
\text { "star", network }\end{array}$ & mobile connection & $\begin{array}{l}\text { mobile } \\
\text { connection }\end{array}$ \\
\hline
\end{tabular}

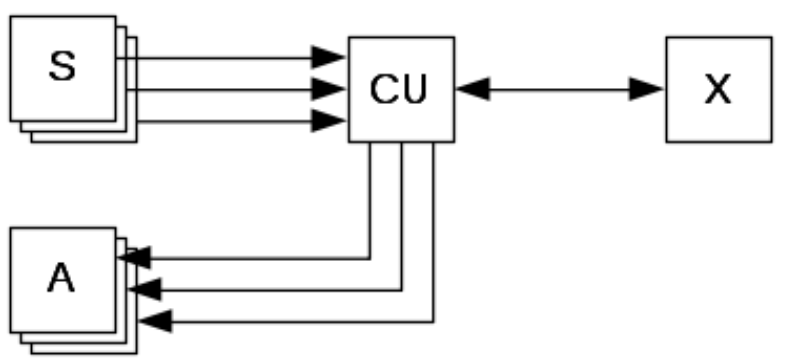

Fig. 1. The generalized scheme of WSAN

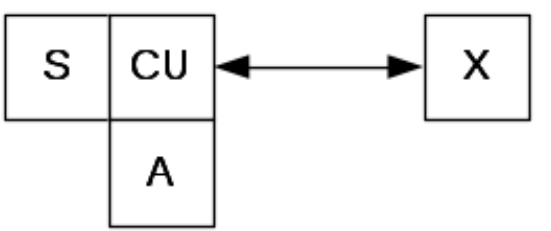

$\mathrm{K}_{1}=[\mathrm{S}, \mathrm{CU}, \mathrm{A}]-[\mathrm{X}]$

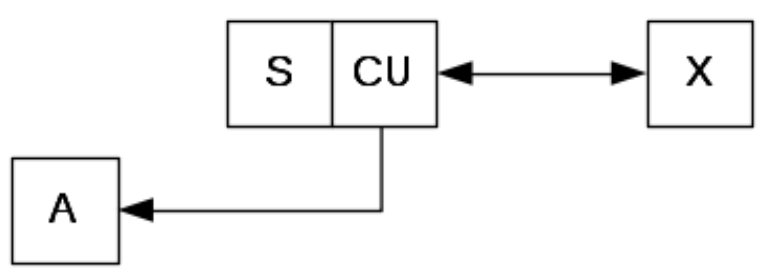

$\mathrm{K}_{3}=[\mathrm{A}]-[\mathrm{S}, \mathrm{CU}]-[\mathrm{X}]$
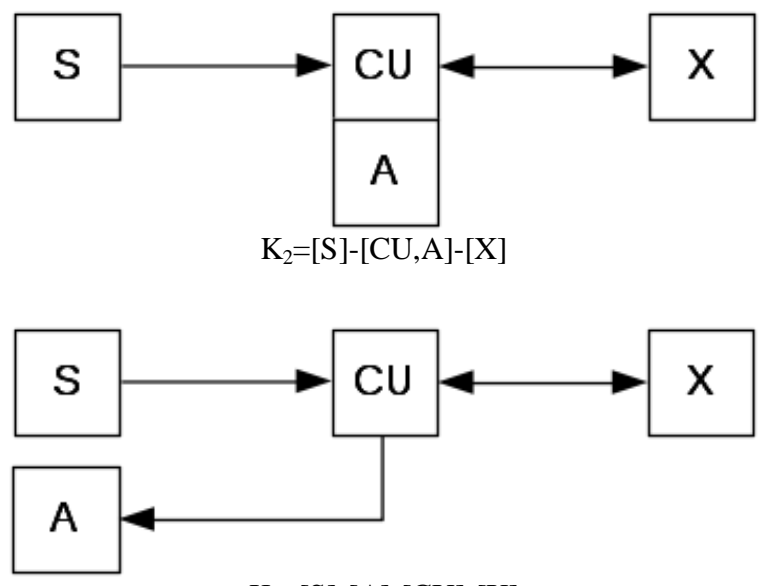

$\mathrm{K}_{4}=[\mathrm{S}]-[\mathrm{A}]-[\mathrm{CU}]-[\mathrm{X}]$

Fig. 2. Variants of the layout of the WSAN nodes

On the basis of this scheme, four basic variants of the layout of the WSAN nodes (Fig. 2) can be defined, that is, the variants of the placement of nodes within one WSAN component (device), in particular: 1) $\mathrm{K}_{1}=[\mathrm{S}, \mathrm{CU}$, $\mathrm{A}]-[\mathrm{X}]$ - sensor node, actuator node and control unit are integrated into one device (and as a result there is only one communication line between the $[\mathrm{S}, \mathrm{CU}, \mathrm{A}]$ and the control console $[\mathrm{X}]$; 2) $\mathrm{K}_{2}=[\mathrm{S}]-[\mathrm{CU}, \mathrm{A}]-[\mathrm{X}]-$ the actuator node and control unit are combined into one device, the sensor node and the control console are separate devices, which means that this variant gives two communication lines; 3) $\mathrm{K}_{3}=[\mathrm{A}]-[\mathrm{S}, \mathrm{CU}]-[\mathrm{X}]-$ the sensor node and control unit are integrated into one device, the actuator node and control unit are separate devices; 4) $\mathrm{K}_{4}=[\mathrm{S}]-[\mathrm{A}]-[\mathrm{CU}]-[\mathrm{X}]-$ all main nodes are implemented as separate devices, which means that this variant gives three communication lines.

So, from the point of view of selecting and implementing given variants of node layout, it is possible to divide all WSANs into 1) homogeneous ones, in which 
only one variant of node layout is implemented, and 2) heterogeneous, in which several layout variants are implemented simultaneously. And in this, the analysis and design of homogeneous WSAN seems to be a simpler task than analyzing and designing heterogeneous WSANs. In the most complex case all major variants of node layout $\left(\mathrm{K}_{1}, \mathrm{~K}_{2}, \mathrm{~K}_{3}, \mathrm{~K}_{4}\right)$ are implemented in the WSAN scheme.

On the basis of one or another variant of the node layout, one can consider different variants of the implementation of communication schemes between WSAN components (Fig. 3).

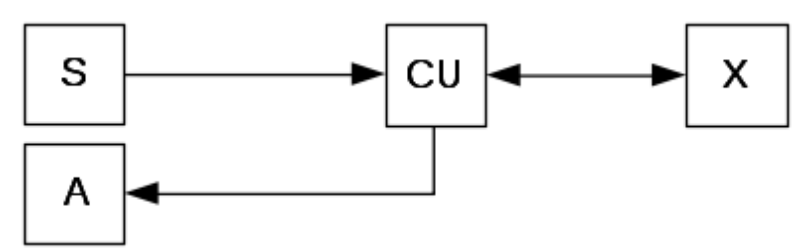

1)

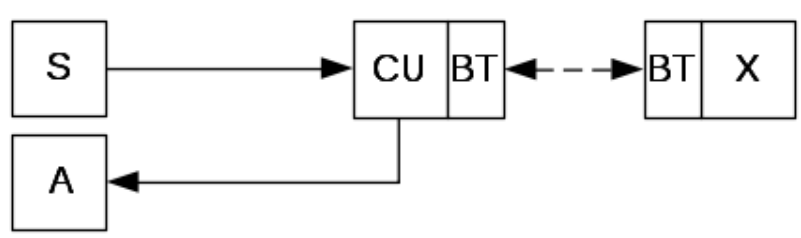

3)

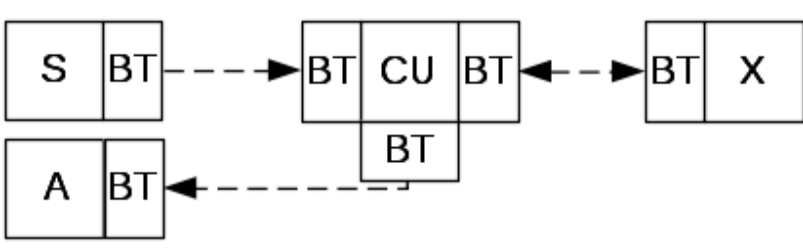

5)

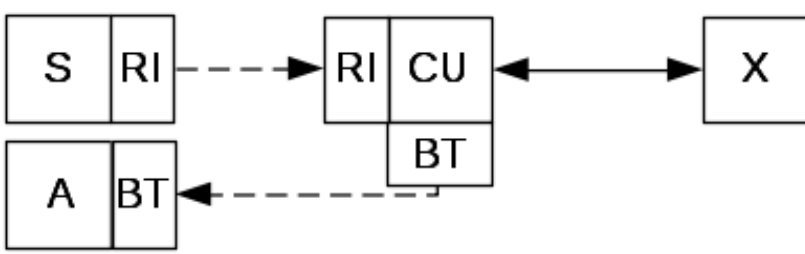

7)

Fig. 3. Variants of the implementation of communication schemes between WSAN components for the node layout variant $K_{4}$

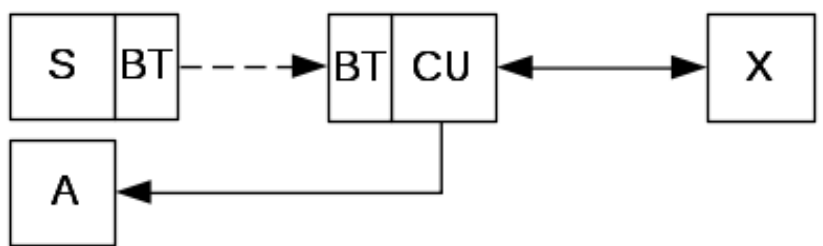

2)

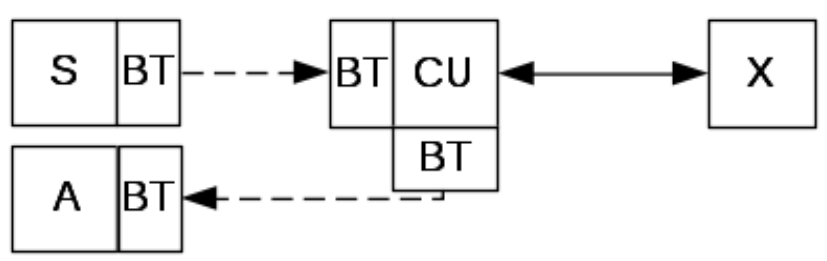

4)

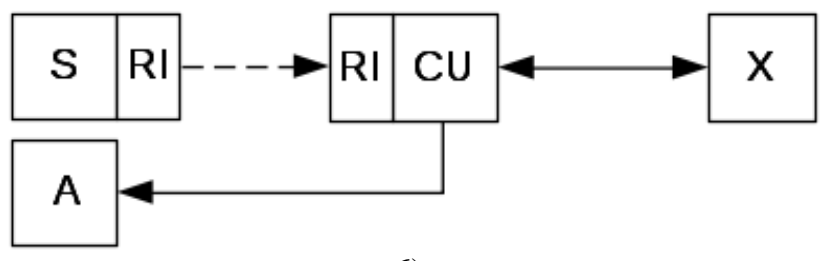

6)

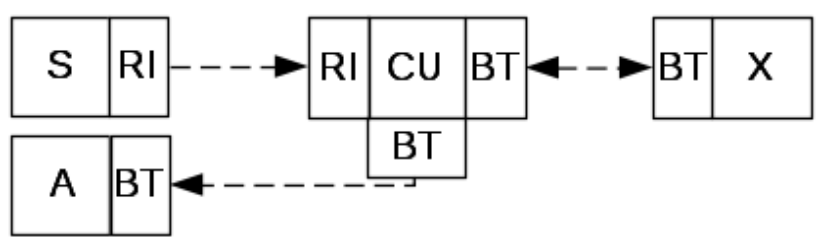

8)

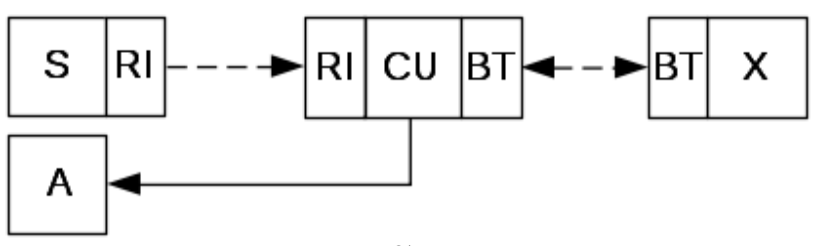

9)

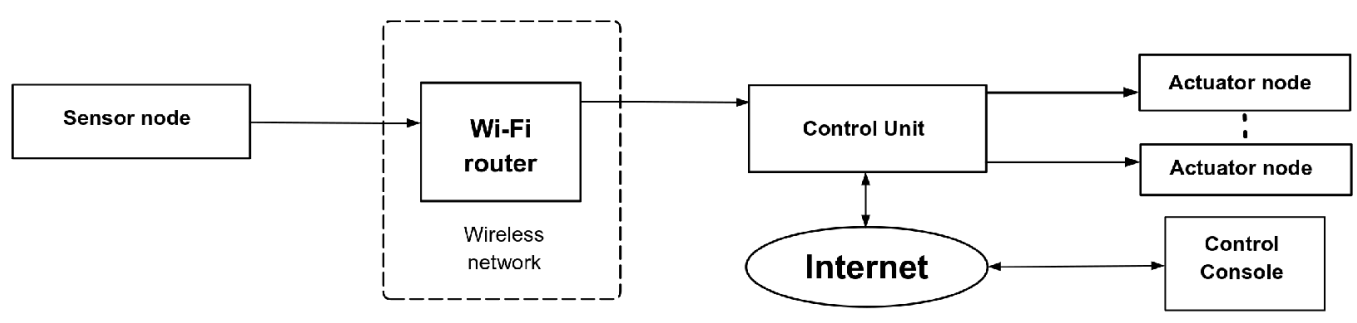

Fig. 4. The overall structure of the WSAN 


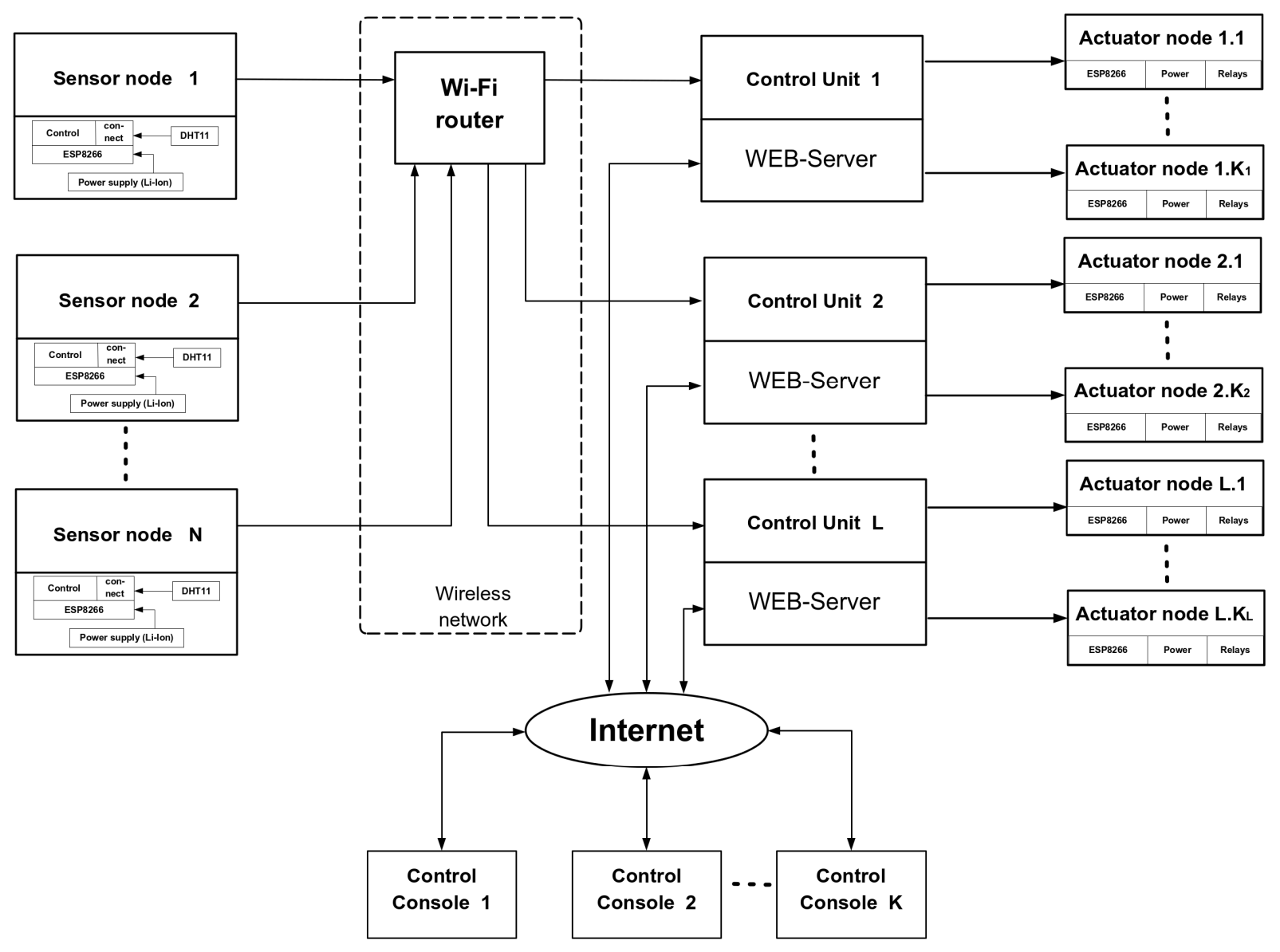

Fig. 5. Detailed structure of the WSAN

Table 2

Characteristics of wireless modules

\begin{tabular}{|c|c|c|c|c|c|c|c|}
\hline № & Module & $\begin{array}{l}\text { Price, } \\
\text { UAH }\end{array}$ & $\begin{array}{c}\text { Power } \\
\text { consumption, } \mathrm{mA}\end{array}$ & Distance, $\mathrm{m}$ & Standard size, $\mathrm{mm}$ & $\begin{array}{l}\text { Bandwidth, } \\
\text { Mbps }\end{array}$ & Remarks \\
\hline & Bluetooth & & & & & & \\
\hline 1 & HC-03-06 & 150 & $30-40$ & 10 & $44 \times 16 \times 4$ & $2-3$ & \\
\hline 2 & HC-07 & 200 & 20 & 10 & $44 \times 16 \times 4$ & $2-3$ & Low Energy \\
\hline 3 & HC-08 & 200 & 20 & 80 & $44 \times 16 \times 4$ & 8 & Low Energy \\
\hline 4 & HC-09 & 170 & 25 & 10 & $44 \times 16 \times 4$ & 8 & $\begin{array}{l}\text { replacement } \\
\text { of HC-06-07 }\end{array}$ \\
\hline & Radio & & & & & & \\
\hline 5 & HM-R433 & 170 & 5 & 200 & $30 \times 14 \times 7$ & 0.004 & $433 \mathrm{MHz}$ \\
\hline 6 & NRF24L01 & 50 & 12.5 & 50 & $29 \times 16 \times 13$ & 2 & $2.4 \mathrm{GHz}$ \\
\hline & $\mathrm{Wi}-\mathrm{Fi}$ & & & & & & \\
\hline 7 & ESP8266 & 120 & 15 & 20 & $11.5 \times 11.5 \times 2$ & 5 & $2.4 \mathrm{GHz}$ \\
\hline
\end{tabular}

For example, for the $\mathrm{K}_{4}$ layout variant and the set of available communication methods $\mathrm{C}=\left\{\mathrm{c}_{1}, \mathrm{c}_{2}, \mathrm{c}_{3}\right\}$, where $\mathrm{c}_{1}$ is a wired connection, $\mathrm{c}_{2}$ is a Bluetooth-based connection (BT), and $c_{3}$ is wireless connection based on simple RF module (RI), it is possible to define nine basic variants of implementation of communication scheme between components of WSAN (Fig. 3), in particular: 1) wired connections between all components; 2) the connection between the sensor node and the control unit via the Bluetooth, other connections are wired; 3) the connection between the control unit and the control console via the Bluetooth, other connections are wired; 4) the connection between the sensor node, the control unit and the actuator node via the Bluetooth interface, other connections are wired; 5) the connection between all components via the Bluetooth; 6) the connection between 
the sensor node and the control unit via the radio interface; other connections are wired; 7) the connection between the sensor node and the control unit via the radio interface, between the actuator node and the control unit via the Bluetooth, the connection between the control console and the control unit is wired; 8) the connection between the

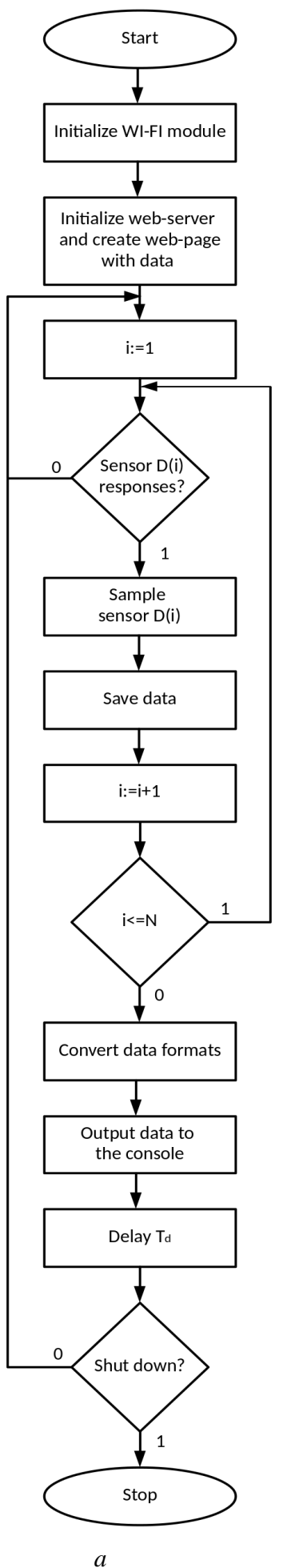

sensor node and the control unit via the radio interface, other connections are through the Bluetooth; 9) the connection between the sensor node and the control unit via the radio interface, between the control unit and the actuator node via a wired connection, between the control console and the control unit via the Bluetooth interface.

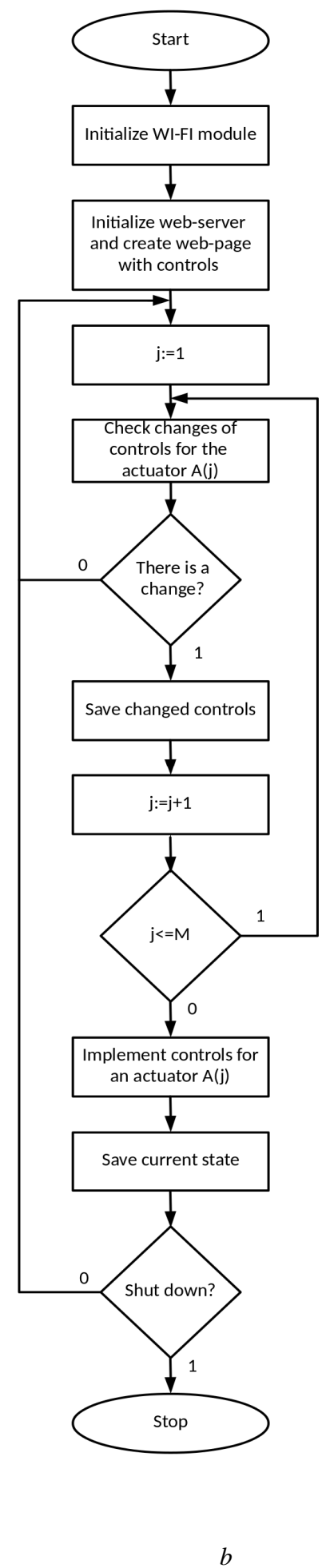

Fig. 6. WSAN algorithms 
Based on the analysis of wireless technologies and modules (Table 2), WSAN node layout variants, and variants of implementing communication schemes between WSAN components, we have selected Wi-Fi technology and, accordingly, the wireless SoC ESP8266 for design of a homogeneous WSAN with the node layout variant $\mathrm{K}_{4}$.

\section{WSAN: STRUCTURE AND ALGORITHMS OF OPERATION}

The basic functionality of WSAN is implemented in the following way (Fig. 4). The control unit takes the measuring information from the sensor nodes through the Wi-Fi interface using a router. This information can be accessed from any control console. The designed structure of WSAN also provides the possibility of autonomous operation of the actuator nodes, which receive the results of the analysis of the measuring information from the control units. In addition one can give commands to the actuator nodes directly from the control console, or let them receive commands from the control units in an autonomous operation mode. Communication between actuator nodes and control units is also carried out via the Wi-Fi.

Thus, the WSAN structure consists of the following main components: sensor nodes, control units, actuator nodes, control consoles, and data transfer medium (Fig. 5). In this case, 1) the sensor node contains sensors, Wi-Fi module (ESP8266) and a lithium-ion battery for autonomous operation; 2) the actuator node contains an Arduino UNO controller for the management of actuators, a Wi-Fi module (ESP8266) and a lithium-ion battery; 3) the data transfer medium is implemented on the basis of one or more Wi-Fi routers with access to the Internet; 4) the possibility of remote access to the WSAN from the control consoles is implemented on the basis of web-servers, which are executed on the corresponding control units.

The sensor node operation algorithm (Fig. 6, a) consists of the following steps. The sensor node initializes its own web-server and creates a corresponding web-page. Next, the sensor node samples its sensors $\{D(i)\}$, reads the measuring information and saves it. When information from all sensors is received, the necessary transformation of data formats and the output of information on the previously created web-page are performed. Then there is a delay and a transition to the beginning of the cycle.

The actuator node operation algorithm (Fig. 6, b) consists of the following steps. The actuator node initializes its own web-server and creates a corresponding web-page. This page displays controls (web-interface buttons) for implementing control commands (for example, switch on or switch off some device). A web-page poll is then conducted for the purpose of detecting the change of the controls. If the change detected, the corresponding actuator will be activated or deactivated, and the status will be saved until the next command arrives.

\section{THE SENSOR NODE DESIGN}

The sensor node design has four main functional modules (Fig. 7): the sensor module, the communication module, the unit of power supply for the communication module, the unit of power supply for the sensor module. The sensor module is implemented using the DHT11 humidity and temperature sensor. The sensor node's communication module is implemented on the basis of the wireless SoC ESP8266 (wireless Wi-Fi module). It reads data from the sensor module, decodes it and transfers it to the control unit. The unit of power supply for the communication module provides $3.3 \mathrm{~V}$ DC voltage using the LM317T stabilizer. In addition, at the input and output of the stabilizer there are capacitors for anti-oscillation (with capacitance from $60 \mathrm{nF}$ to $100 \mathrm{nF}$ ). The unit of power supply for the sensor module provides $5 \mathrm{~V}$ DC voltage using the NCP1117S stabilizer and capacitors at its output to smooth the oscillations.

The design of the prototype of the sensor node consists of four components (Fig. 8) in the case with dimensions of $80 \times 60 \times 30 \mathrm{~mm}$ (Fig. 9). The case has openings for the switch and the micro-USB connector used to charge the battery. The main characteristics of the implemented prototype of the sensor node are as follows: 1) power supply $-3.7 \mathrm{~V} ; 2$ ) power consumption $-82 \mathrm{~mW}$; 3) battery life (in constant operation mode) - 96 hours; 4) the maximum number of sensors - 2; 5) battery capacity $-2400 \mathrm{~mA}$; 6) full charge time -2.5 hours; 7) overall dimensions $-80 \times 60 \times 30 \mathrm{~mm}$.

\section{THE ACTUATOR NODE DESIGN}

The actuator node design has six main functional modules (Fig. 10): the microcontroller, the communication module, the unit of power supply for the communication module, the unit of power supply for the microcontroller, the synchronization unit, the reset unit. The ATMEGA328-A based on the Arduino Uno board is used as a microcontroller. The microcontroller manages the communication module and the actuators using the relays. The actuator node's communication module is also implemented on the basis of the wireless SoC ESP8266. It is used to transmit and receive data, in particular to create a web-server and a web-page with control elements on it, as well as to monitor the state changes of these elements. The synchronization unit generates clock signals for a microcontroller using the quartz with frequency $32.768 \mathrm{KHz}$ (connected to pins XTAL1 and XTAL2 of the microcontroller). The reset unit generates a reset signal for the initial initialization of the microcontroller (connected to the pin RST of the microcontroller).

The design of the prototype of the actuator node consists of three components (Fig. 11) in the case with dimensions of $90 \times 65 \times 35 \mathrm{~mm}$ (Fig. 11). The case has openings for the Arduino Uno jacks and power contacts of the relays. The main characteristics of the implemented prototype of the actuator node are as follows: 1) the maximum number of devices under the control of one actuator node -11 ; 2) maximum power of the actuator $2 \mathrm{~kW}$; 3) power consumption - $1.25 \mathrm{~W}$; 4) overall dimensions $-90 \times 65 \times 35 \mathrm{~mm}$. 

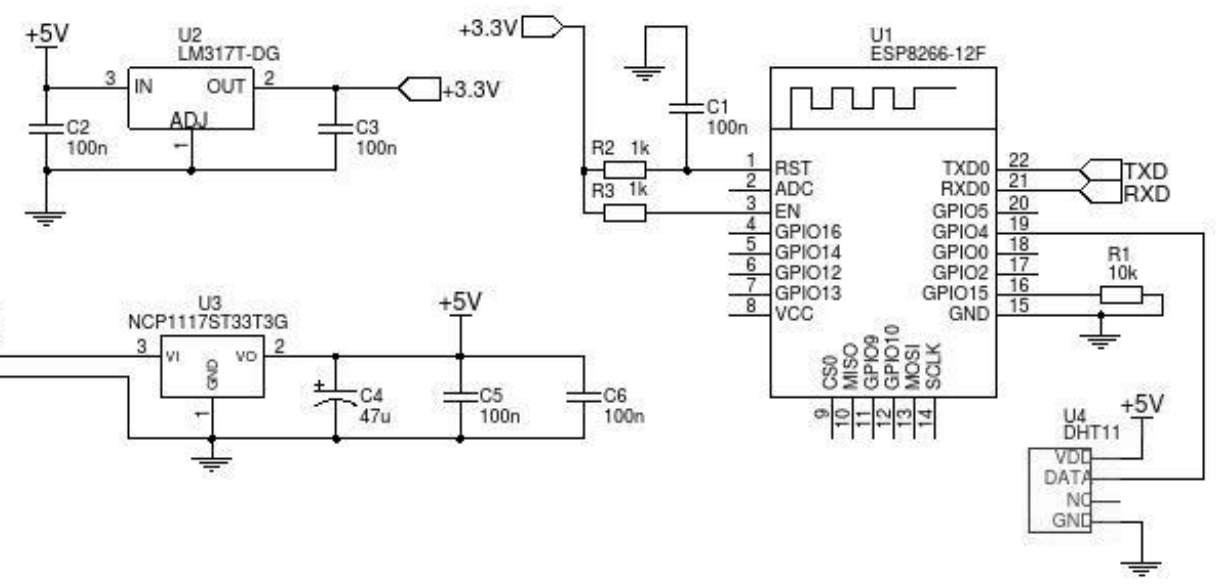

Fig. 7. Functional diagram of the sensor node

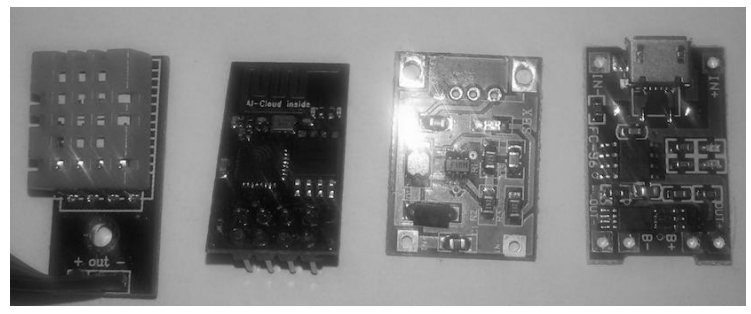

Fig. 8. Components of the prototype of the sensor node

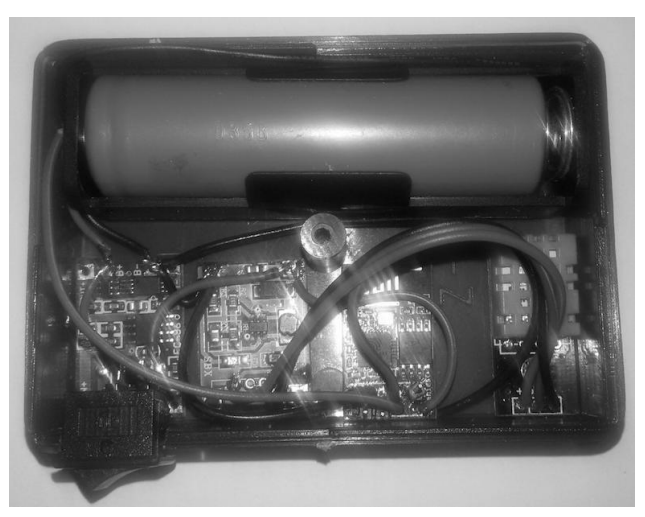

Fig. 9. The design of the prototype of the sensor node
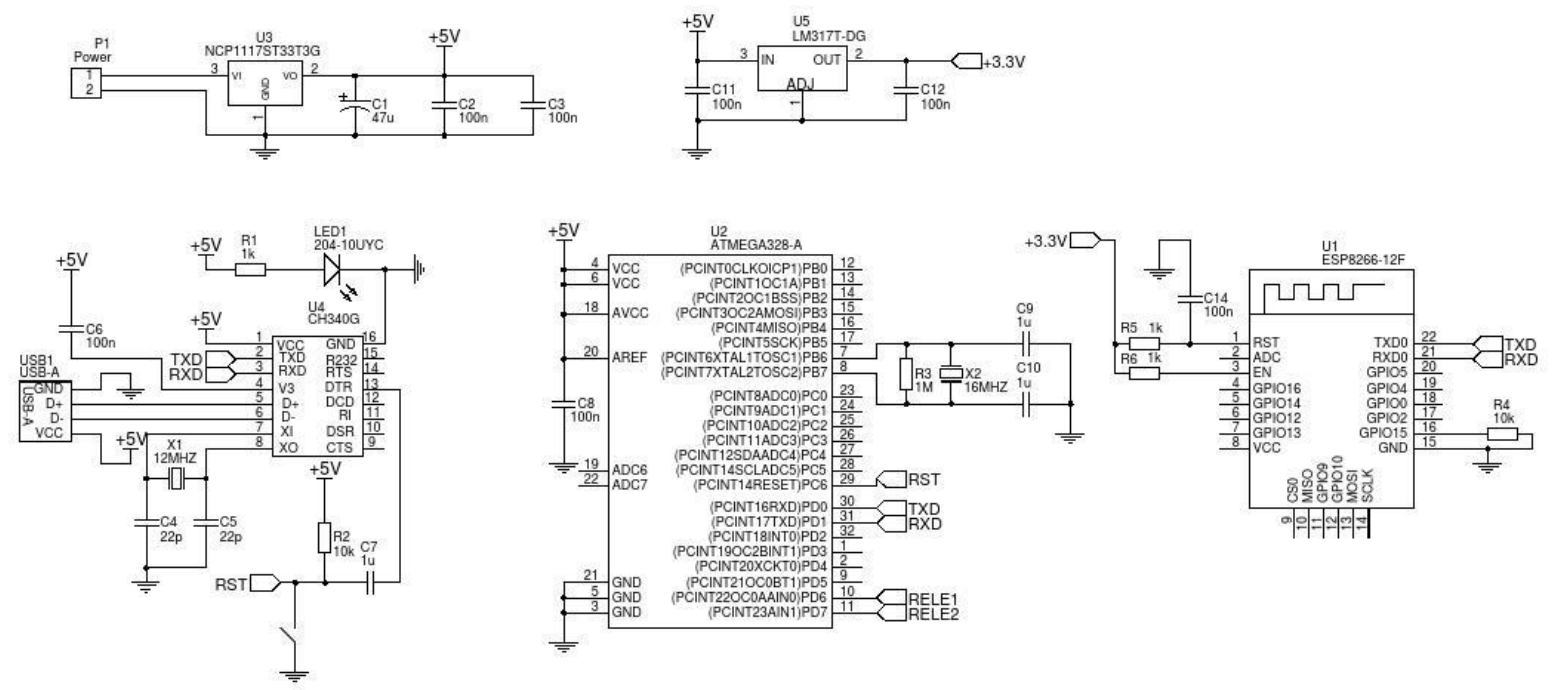

Fig. 10. Functional diagram of the actuator node 


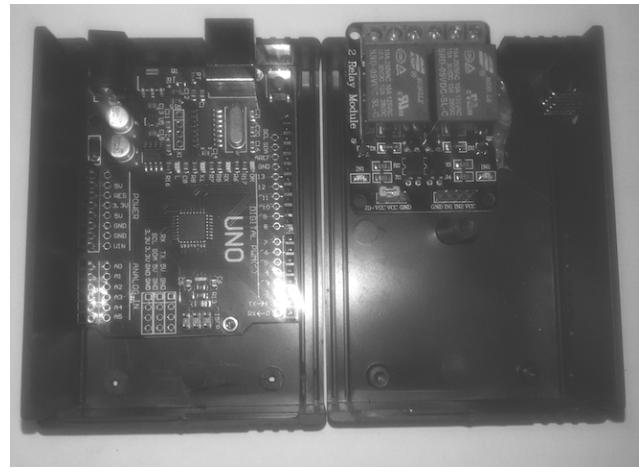

Fig. 11. The design of the prototype of the actuator node

\section{CONCLUSIONS}

The problem of wireless sensor-actuator network design as a part of the mobile cyber-physical system was considered. Various variants of WSAN node layout, various variants of wireless communication technologies for its construction and methods of implementation of the wireless communication scheme are analyzed. The WSAN structure and algorithms of its operation based on the Wi-Fi technology are proposed. The design of the sensor and actuator nodes based on the wireless $\mathrm{SoC}$ ESP8266 is presented. The basic functionality of the designed WSAN includes: 1) the ability to collect measuring information from the sensors of different physical nature and execute commands by the actuators in automatic mode; 2) the ability to remotely view the sensor readings and give commands for the actuators from the control consoles on mobile computing devices.

The results presented in this paper were obtained within the framework of the research project DB/CIBER (registration number 0115U000446), 01.01.2015 31.12.2017, funded by the Ministry of Education and Science of Ukraine.

\section{REFERENCES}

[1] Melnyk A., Cyber-physical systems: problems of creation and directions of development, Transactions on Computer systems and networks, Lviv Polytechnic National University Press, No. 806, 2014. - pp. 154-161 (in Ukrainian)

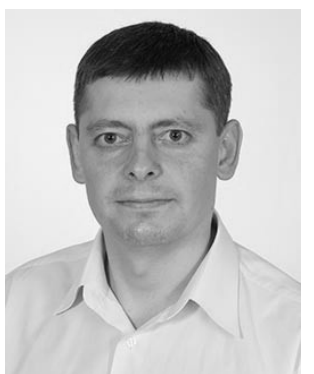

Alexey Botchkaryov was born in 1975 in Lviv, Ukraine. He received the B.S. and the M.S. degrees in computer engineering from Lviv Polytechnic Natioinal University in 1996 and 1998. He has been doing scientific and research work since 1994. His work resulted in 79 publications and master thesis. Currently, he is a Seniour Lecturer with the Computer Engineering Department and a Research Assistant with the Intelligent Systems Laboratory, Lviv Polytechnic Natioinal University. His research interests include intelligent data collection technologies and multi-agent systems.
[2] Melnyk A., Integration of the levels of the cyber-physical system, Transactions on Computer systems and networks, Lviv Polytechnic National University Press, No. 830, 2015. - pp. 61-67 (in Ukrainian)

[3] Golembo V., Botchkaryov A., Approaches to the construction of conceptual models of cyber-physical systems, Transactions on Computer Science and Information Technology, Lviv Polytechnic National University Press, No. 864, 2017. - pp. 168-178 (in Ukrainian)

[4] Rajeev Alur, Principles of Cyber-Physical Systems, The MIT Press, 2015. - 464 p.

[5] Siddhartha Kumar Khaitan and James D. McCalley, Design Techniques and Applications of CyberPhysical Systems: A Survey, IEEE Systems Journal, Volume: 9, Issue: 2, June 2015. pp. $350-365$.

[6] Jules White, Siobhan Clarke, Christin Groba, Brian Dougherty, Chris Thompson, Douglas C. Schmidt, R\&D challenges and solutions for mobile cyber-physical applications and supporting Internet services, Journal of Internet Services and Applications, May 2010, Volume 1, Issue 1, pp. 45-56.

[7] Boyko Yu. M., Lokazyuk V. M., Mishan V. V. Conceptual characteristics of the implementation of wireless sensor networks, Transactions of Khmelnytsky National University, No. 2, 2010.pp. 94-97 (in Ukrainian)

[8] Wireless Sensor and Actuator Networks: Algorithms and Protocols for Scalable Coordination and Data Communication, ed. by Amiya Nayak and Ivan Stojmenovic, John Wiley \& Sons, 2010. $-350 \mathrm{p}$.

[9] Chenyang Lu, Abusayeed Saifullah, Bo Li, Mo Sha, Humberto Gonzalez, Dolvara Gunatilaka, Chengjie Wu, Lanshun Nie, Yixin Chen, Real-Time Wireless Sensor-Actuator Networks for Industrial Cyber-Physical Systems, Proceedings of the IEEE, Volume 104 Issue 5, 2016. - pp. 1013-1024

[10] Botchkaryov A., Golembo V., Applying intelligent technologies of data collection to autonomous cyber-physical systems, Transactions on Computer systems and networks, Lviv Polytechnic National University Press, No. 830, 2015. - pp. 7-11 (in Ukrainian)

[11] Melnyk A., Golembo V., Botchkaryov A., The new principles of designing configurable smart sensor networks based on intelligent agents, Transactions on Computer systems and networks, Lviv Polytechnic National University Press, No. 492, 2003. pp. 100-107 (in Ukrainian)

[12] Botchkaryov A., Collective behavior of mobile intelligent agents solving the autonomous distributed exploration task, Transactions on Computer systems and networks, Lviv Polytechnic National University Press, No. 546, 2005. - pp. 12-17 (in Ukrainian)

[13] Botchkaryov A., Structural adaptation of the autonomous distributed sensing and computing systems, Transactions on Computer systems and networks, Lviv Polytechnic National University Press, No. 688, 2010. - pp. 16-22 (in Ukrainian)

[14] Botchkaryov A., The problem of organizing adaptive sensing and computing processes in autonomous distributed systems, Transactions on Computer systems and networks, Lviv Polytechnic National University Press, No. 745, 2012. - pp. 20-26 (in Ukrainian)

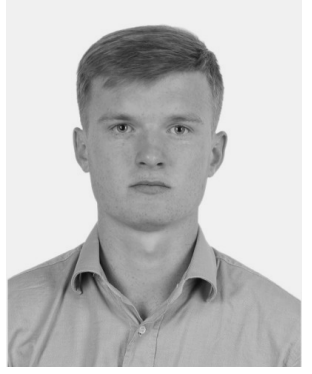

Yuriy Kraikin was born in 1995 in Lviv, Ukraine. He received the B.S. degree in computer engineering from the Lviv Polytechnic Natioinal University in 2017. He has been doing scientific and research work since 2014. Currently, he is a graduate student of the Computer Engineering Deprtment at Lviv Polytechnic Natioinal University. His research interests include wireless sensor-actuator networks and cyberphysical systems. 